\title{
CARGA DE ENFERMEDAD POR TABAQUISMO E IMPACTO POTENCIAL DEL INCREMENTO DE PRECIOS DE CIGARRILLOS EN EL PERÚ
}

\author{
Ariel E. Bardach 1,2,a, Joaquín E. Caporale 1,b , Andrea Alcaraz ${ }^{1, c}$, Federico Augustovski 1,2,3,a,

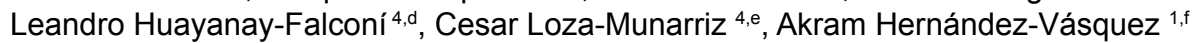 \\ Andrés Pichon-Riviere ${ }^{1,2,3, a}$
}

\begin{abstract}
RESUMEN
Objetivos. Estimar la carga de enfermedad asociada al tabaquismo y evaluar el potencial impacto económico y en salud del aumento de los impuestos a los cigarrillos en el Perú. Materiales y métodos. Mediante un modelo de microsimulación se estimó el impacto en mortalidad, calidad de vida y costos atribuibles al tabaquismo por enfermedad cardiaca y cerebrovascular, enfermedad pulmonar obstructiva crónica, neumonía, cáncer de pulmón y otras nueve neoplasias. Se evaluaron tres escenarios de aumento de impuestos. Resultados. Un total anual de 16719 muertes, 6926 diagnósticos de cáncer, 7936 accidentes cerebrovasculares y 7548 hospitalizaciones por enfermedad cardiovascular se pueden atribuir al tabaquismo en Perú. Asimismo, se pierden 396069 años de vida por muerte prematura y discapacidad cada año, y el costo de tratar los problemas de salud ocasionados por el tabaco asciende a 2500 millones de soles (PEN 2015). Actualmente, los impuestos al tabaco llegan a cubrir solamente el $9,1 \%$ de dicho gasto. Un incremento del $50 \%$ en el precio de los cigarrillos podría evitar 13391 muertes, 6210 eventos cardiovasculares y 5361 nuevos cánceres en los próximos diez años, y representaría un beneficio económico de 3145 millones (PEN) por ahorro de costos sanitarios y aumento de la recaudación impositiva. Conclusiones. La carga de enfermedad y el costo para el sistema de salud asociados al tabaquismo son elevados en Perú. Incrementar los impuestos al cigarrillo podría derivar en importantes beneficios para el país, tanto sanitarios como económicos.
\end{abstract}

Palabras clave: Uso de tabaco; Costo de enfermedad; Análisis costo-beneficio; Impuestos; Perú (fuente: DeCS BIREME).

\section{BURDEN OF SMOKING-RELATED DISEASE AND POTENTIAL IMPACT OF CIGARETTE PRICE INCREASE IN PERU}

\begin{abstract}
Objectives. To calculate the burden of smoking-related disease and evaluate the potential economic and health impact of tax-induced cigarette price increase in Peru. Materials and methods. A microsimulation model was used to estimate smoking-attributable impact on mortality, quality of life, and costs associated with heart and cerebrovascular disease, chronic obstructive pulmonary disease, pneumonia, lung cancer, and another nine cancers. Three scenarios, involving increased taxes, were evaluated. Results. A yearly total of 16,719 deaths, 6,926 cancer diagnoses, 7,936 strokes, and 7,548 hospital admissions due to cardiovascular disease can be attributed to smoking in Peru. Similarly, 396,069 years of life are lost each year from premature death and disability, and the cost of treating smoking-attributable health issues rises to 2,500 million soles (PEN 2015). Currently, taxes on tobacco cover only $9.1 \%$ of this expense. If cigarette prices were to increase by $50 \%$ over the next 10 years, 13,391 deaths, 6,210 cardiovascular events, and 5,361 new cancers could be prevented, representing an economic benefit of 3,145 million (PEN) in savings in health costs and increases in tax revenues. Conclusions. Smoking-attributable burden of disease and costs to the health system are very high in Peru. Higher cigarette taxes could have substantial health and economic benefits for the country.
\end{abstract}

Key words: Tobacco use; Cost of illness; Cost-Benefit analysis; Taxes; Peru (source: MeSH NLM).

1 Instituto de Efectividad Clínica y Sanitaria (IECS). Buenos Aires, Argentina.

Consejo Nacional de Investigaciones Científicas y Técnicas (CONICET). Buenos Aires, Argentina.

Escuela de Salud Pública, Facultad de Medicina, Universidad de Buenos Aires (UBA). Buenos Aires, Argentina.

4 Unidad de Epidemiología Clínica, Facultad de Medicina Alberto Hurtado, Universidad Peruana Cayetano Heredia. Lima, Perú.

a Médico, doctor en Salud Pública; ${ }^{b}$ licenciado en Economía, magíster en Finanzas Públicas Provinciales y Municipales; ${ }^{c}$ médica especialista en Cardiología, magíster en Efectividad Clínica; ${ }^{\mathrm{d}}$ médico especialista en Medicina Interna, magíster en Epidemiología Clínica; ${ }^{\mathrm{e}}$ médico especialista en Nefrología; ${ }^{\mathrm{f}}$ médico, magíster en Gestión y Políticas Públicas.

Recibido: 18/03/2016 Aprobado: 12/10/2016

Citar como: Bardach AE, Caporale JE, Alcaraz A, Augustovski F, Huayanay-Falconí L, Loza-Munarriz C, et al. Carga de enfermedad por tabaquismo e impacto potencial del incremento de precios de cigarrillos en el Perú. Rev Peru Med Exp Salud Publica. 2016;33(4):651-61. doi: 10.17843/rpmesp.2016.334.2548 


\section{INTRODUCCIÓN}

A nivel global, el tabaquismo es una de las principales causas de enfermedad y muerte evitable, se le atribuye el $6,3 \%$ de las muertes prematuras y años de vida ajustados a discapacidad ocurridos en el mundo entre 1990 y $2010^{(1)}$. En Sudamérica ocupa el tercer lugar dentro de los factores de riesgo de muertes y años de vida saludables perdidos (1), generando baja productividad y aumento del gasto de bolsillo que afecta la economía de los individuos (2), así como de altos costos para los sistemas de salud ${ }^{(3)}$. En Perú, la prevalencia del tabaquismo fluctúa entre 19 y $36 \%$ en hombres y entre 10 a $18 \%$ en mujeres ${ }^{(4)}$; sin embargo, algunos estudios en áreas periurbanas de Lima muestran menores prevalencias ${ }^{(5)}$.

Con el objetivo de controlar el consumo de tabaco, la Organización Mundial de la Salud (OMS) impulsó el Convenio Marco para el Control del Tabaco (CMCT) suscrito y ratificado en la actualidad por la mayoría de países de la región, incluido el Perú (6). Es así que, como parte del CMCT, el gobierno peruano promulgó en el 2006 la Ley 28705, que establecía, entre otros, la impresión de advertencias visuales en el $50 \%$ de una cara de la cajetilla. Complementariamente, el 2010 se promulgó la Ley 29517 que prohíbe el consumo de tabaco en todo lugar de trabajo y lugares públicos. Si bien estas leyes representan un avance, no se consideró ninguna medida tributaria (enmarcada en el artículo 6 del CMCT), y se mantiene hasta la fecha la falta de políticas tributarias y de precios recomendadas por el Convenio.

El aumento del precio de cigarrillos a través de impuestos ha demostrado ser una medida muy costo-efectiva para el control del tabaquismo ${ }^{(3,7)}$. Con ello, se alentaría al consumidor a dejar de fumar, aumentando el número y éxito de los intentos ${ }^{(8)}$, previniendo el inicio en jóvenes, desalentando a exfumadores a reincidir y disminuyendo el consumo entre los fumadores ${ }^{(9,10)}$ con la consecuente reducción de carga de enfermedad y costos sanitarios. Sin embargo, en el Perú, la carga tributaria al tabaco es de las más bajas en Latinoamérica alcanzando un $39,49 \%$ del costo promedio por paquete de 20 unidades (USD 3,70); asimismo, los precios de cigarrillos son bajos comparados con otros países de la región ${ }^{(11)}$, lo cual facilita su acceso; por tal motivo, la OMS recomienda a los gobiernos incrementar los precios del tabaco.

Bajo este escenario, existe un amplio margen para el aumento del precio de los cigarrillos a través de impuestos en Perú y se esperaría que este aumento obtenga externalidades positivas en salud y mejoras en la recaudación; no obstante, a pesar de la importancia de estas medidas, no se han encontrado publicaciones que incorporen modelos económicos para estimar su impacto en el país.

En tal sentido, el objetivo de nuestro estudio fue estimar la carga de enfermedad asociada al tabaquismo y evaluar el impacto económico y en salud que podrían tener el incremento de los impuestos a los cigarrillos en el Perú.

\section{MATERIALES Y MÉTODOS}

Se utilizó un modelo de decisión con dos módulos principales. Uno desarrollado dentro de un proyecto que tuvo la participación de más de 40 investigadores y decisores en universidades, centros de investigación y agencias sanitarias de Argentina, Bolivia, Brasil, Chile, Colombia, México y Perú, el cual ha sido validado y aplicado en estudios que estimaron la carga de enfermedad por tabaquismo y costo-efectividad de intervenciones para control del tabaquismo en Latinoamérica. Asimismo, se desarrolló otro módulo para complementar la evaluación del impacto en salud y calcular la recaudación impositiva ante tres escenarios de incremento de precios de cigarrillos.

\section{MÓDULO DE CARGA DE ENFERMEDAD}

Su desarrollo incluyó una microsimulación de Monte Carlo de primer orden (simulación probabilística de individuos) programado en Microsoft Excel ${ }^{\circledR} 2003$ (Microsoft, Redmond, WA) con macros en Microsoft Visual Basic® 6.3 (Microsoft, Redmond, WA). El módulo incorpora la historia natural, costos y calidad de vida de patologías relacionadas con el tabaquismo en adultos: enfermedad cardíaca coronaria y no coronaria, accidente cerebrovascular (ACV), enfermedad pulmonar obstructiva crónica (EPOC), neumonía, cáncer de pulmón y otras nueve neoplasias (boca y faringe, esófago, estómago, páncreas, riñón, laringe, vejiga, cuello y leucemia) para calcular el riesgo basal de los no fumadores por edad y sexo en cada condición de salud. Una descripción detallada del mismo puede encontrarse en diversas publicaciones que utilizaron o evaluaron el módulo (11,12-17).

El seguimiento de los individuos se realiza en seis cohortes simuladas (tres para hombres y tres para mujeres de cinco millones cada una) de 10000000 de fumadores, 10000000 de exfumadores y 10000000 de no fumadores, desde los 35 años hasta la muerte, estimándose un riesgo individual de ocurrencia para cada evento, de progresión de enfermedad o de muerte. Se incluyeron atributos demográficos, condición de fumador, estado clínico y las ecuaciones de riesgo subyacentes, que permiten obtener resultados agregados en términos de salud y costos. Las incidencias se estimaron a partir 
de la mortalidad y letalidad de cada condición en eventos agudos ante la falta de datos de buena calidad. Para neoplasias y otras patologías crónicas, se estimaron las probabilidades de diagnóstico por edad y sexo, mediante una aproximación de las tasas de mortalidad anual de registros nacionales y de la sobrevida anual estimada desde el diagnóstico.

Para incluir las consecuencias del tabaquismo pasivo, se incorporó la estimación de muertes, años de vida y costos asociados a esta condición a partir de una carga adicional del $13,6 \%$ en hombres y del $12,0 \%$ que ha sido estimada en otros estudios ${ }^{(18)}$.

El módulo permite calcular como desenlaces las muertes y los eventos de salud asociados a enfermedades relacionadas al tabaquismo. Asimismo, estima los años de vida perdidos por muerte prematura, así como por discapacidad o reducción de calidad de vida. Es posible estimar los años totales de vida ajustados por calidad (AVAC).

\section{CALIBRACIÓN Y VALIDACIÓN}

Los procedimientos seguidos incluyeron: 1) Consistencia interna, para identificar errores relacionados con la incorporación de datos y sintaxis de modelado; 2) Calibración, para garantizar la reproducibilidad de los resultados en indicadores de incidencia y mortalidad, y 3) Validación externa, donde los resultados se contrastan con estudios no incluidos en la estimación.

El tamaño muestral se estimó en base al error estándar del parámetro con mayor variabilidad (incidencia de cáncer oral) para obtener intervalos de confianza al $95 \%$ en el rango de $+/-2,5 \%$ en cada una de las seis cohortes. Las tasas de mortalidad para cada una de las condiciones incluidas en el módulo se compararon con las tasas de mortalidad para esas mismas condiciones reportadas en las estadísticas nacionales, mostrando una excelente validez interna.

\section{FUENTES DE INFORMACIÓN}

Los datos incluidos fueron obtenidos mediante una revisión de la literatura en múltiples bases de datos electrónicas, incluyendo MEDLINE, EMBASE, CENTRAL, SocINDEX, EconLit, LILACS, NBER, CRD y Grupo de revisión de tabaco de Cochrane. Además, se realizó una búsqueda de literatura gris para identificar información relevante de ministerios de salud, ministerios de finanzas, OMS y de congresos. Los investigadores participantes proporcionaron información adicional de encuestas, estadísticas vitales y bases de datos de egresos hospitalarios.

\section{COSTOS DE ATENCIÓN MÉDICA}

Se definió una metodología de costeo mixta contemplando la posibilidad de estimar los costos de los eventos mediante un enfoque de micro o macrocosteo, de los 15 eventos de salud incluidos en el módulo.

El cáncer de pulmón (primer año), el seguimiento anual coronario y ACV (primer año), fueron valuados siguiendo un enfoque de microcosteo. El costo del cáncer de pulmón (primer año) corresponde a la estimación del protocolo de atención del Instituto Nacional de Enfermedades Neoplásicas (INEN) y el seguimiento (segundo año y subsiguientes) se valuó relacionando su mismo peso relativo sobre el costo del primer año en Argentina. Algunos costos se estimaron utilizando una proporción aplicada sobre el PIB per cápita en moneda local, representada por el peso de los costos de los eventos verificados en Argentina ${ }^{(15)}$, Chile ${ }^{(16)}$ y México ${ }^{(17)}$ sobre el PIB per cápita en moneda local teniendo como fuente de información el Banco Mundial (http://data.worldbank.org). Los eventos costeados de esta manera fueron: infarto agudo de miocardio (IAM) (primer y segundo año), evento coronario (no IAM), seguimiento del $A C V$, neumonía y EPOC.

Asimismo, los costos de los tipos de cáncer restantes (excepto el de pulmón) se estimaron en referencia a la estimación realizada para el primer año y segundo año del cáncer de pulmón. Para ello se utilizó un método de consenso de expertos con metodología Delphi modificada para estimar la relación existente entre cada uno de los costos totales para la atención de cada cáncer y el costo médico directo del cáncer de pulmón.

Se consideró en la identificación y cantidades de recursos los registros de utilización del Hospital Nacional Cayetano Heredia y la Clínica San Felipe en Lima. Los resultados se ponderaron por sector, $90 \%$ sector público y $10 \%$ sector privado, contemplando de esta manera una perspectiva desde el sistema del sistema de salud del Perú. Finalmente, las estimaciones fueron ajustadas según inflación, empleando el índice de precios al consumidor (IPC) del Instituto Nacional de Estadística e Informática (INEI) para expresar los costos y resultados en moneda local (PEN) del 2015.

\section{MÓDULO DE IMPACTO DE POLÍTICAS IMPOSITIVAS}

El impacto del incremento en los impuestos a cigarrillos fue analizado en tres niveles: 1) Impacto en prevalencia de tabaquismo y eventos sanitarios; 2) Impacto en la recaudación impositiva del país, y 3) Impacto financiero total a nivel país. Se consideraron como parámetros claves del módulo: la elasticidad del precio de la demanda, variación en consumo y diferencias de precios. 


\section{IMPACTO EN LA PREVALENCIA DE TABAQUISMO Y EVENTOS EN SALUD}

El impacto esperado para los escenarios de incremento de impuestos a los cigarrillos se incorporó a través de la siguiente ecuación:

Prevalencia ${ }_{\text {post }}=$ Prevalencia ${ }_{\text {pre }}+\left(\left.E_{d}{ }_{d} \Delta \% P^{*}\right|_{p}{ }^{*}\right.$ Prevalencia $\left.{ }_{\text {pre }}\right)$

Donde: Prevalencia $a_{\text {pre }}=$ prevalencia de fumadores previo al incremento de precio; $\mathrm{E}_{\mathrm{d}}=$ elasticidad precio de la demanda; $\Delta \% P=$ diferencia porcentual del precio; e $I_{p}=$ proporción de la variación en el consumo que impacta en la prevalencia de tabaquismo.

Ante la heterogeneidad reportada en la literatura, de la traslación del aumento de impuestos al impacto en el consumo y riesgo, se analizaron tres escenarios -del más conservador al más optimista- para estimar el impacto sanitario:

Escenario de corto plazo: asume que un $50 \%$ de la reducción del consumo impacta en la prevalencia $\left(I_{p}=0,5\right)$ y esta reducción se traslada a la población de exfumadores.

Escenario de mediano plazo: incorpora, además de lo anterior, a los potenciales efectos asociados con reducir el número de cigarrillos consumidos. Siendo un tema controversial, esta reducción de riesgo varía para las diferentes condiciones de salud $(19,20)$. Se asume en este escenario que una reducción del consumo involucrará una reducción del exceso de riesgo del consumidor. Esta reducción fue aplicada en forma proporcional a la reducción en el consumo, al $75 \%$ del exceso de riesgo que separa a un fumador de un exfumador, asumiendo que un $25 \%$ del riesgo adicional, solo se eliminaría cuando el sujeto se transforma en exfumador. Considerar $75 \%$ para el máximo beneficio que podría obtener un fumador al reducir su consumo está basado en la máxima diferencia de riesgo existente entre fumadores de alta y baja intensidad respecto a un exfumador para cáncer de pulmón (82\%), enfermedad cardíaca isquémica $(57 \%)$, y EPOC $(80 \%){ }^{(19)}$.

Escenario a largo plazo: de máximo efecto y que podría esperarse en el largo plazo (ej. 10 años). Similar al escenario anterior, pero con mayor impacto en la prevalencia $\left(I_{p}=0,75\right)$ y en la cual la población de exfumadores se mantiene constante respecto al escenario basal ya que toda la reducción en la prevalencia se traslada a la población de no fumadores. Para estimar el caso base, se combinaron los resultados de estos tres escenarios (desde el inicio de la política hasta los diez años) del siguiente modo: del año 1 al año 5 se asume una evolución lineal del escenario de corto plazo al escenario de mediano plazo; del año 6 al 10 una evolución lineal de mediano al de largo plazo.
Basados en los cambios en prevalencia y redistribución de la proporción de fumadores, exfumadores y no fumadores en la población, se estimó nuevamente la carga de enfermedad bajo los nuevos escenarios, siguiendo la misma metodología de la estimación basal, calculando el impacto como la diferencia entre ambas estimaciones según número de muertes, eventos, años de vida, discapacidad y costos sanitarios.

\section{IMPACTO EN LA RECAUDACIÓN IMPOSITIVA}

Finalmente, los beneficios impositivos se estiman con la siguiente fórmula:

$$
\Delta \mathrm{R}=(1+\Delta \% \text { Consumo }) *\left(1+\frac{\Delta \% \text { Precio }}{\% \text { impuesto }}\right)-1
$$

Donde: $\Delta \mathrm{R}=$ variación en la recaudación impositiva; $\Delta \%$ Consumo $=$ variación porcentual del consumo esperable por el aumento de precio de venta; $\Delta \%$ Precio= cambio porcentual en el precio de venta al público; y $\%$ impuesto= proporción del precio inicial de venta al público que corresponde a impuestos.

\section{IMPACTO ECONÓMICO TOTAL}

Fue estimado mediante la variación entre el gasto inicial directo en salud atribuible al tabaquismo calculado y la variación en la recaudación tributaria.

\section{RESULTADOS}

Los principales parámetros demográficos, epidemiológicos, de costos e impositivos que se incluyen en el modelo se resumen en la Tabla 1.

La correlación entre los resultados observados y los esperados produjo un alto grado de correlación con valores de $R^{2}$ entre 0,700 y 0,999 . La validación externa se realizó comparando los resultados del modelo con estudios que no habían sido utilizados como fuentes de datos, observándose una muy buena correlación entre los resultados predichos y los observados en los estudios (Figura 1).

\section{MUERTES Y EVENTOS ATRIBUIBLES AL TABAQUISMO}

Se estimó un total de 54301 muertes por causas asociadas al tabaquismo, de las cuales el $31 \%$ son atribuibles al tabaquismo en el 2015 (Tabla 2). El 17\% de las muertes cardiovasculares y el $17 \%$ de las producidas por ACV pueden atribuirse al tabaquismo. La mortalidad es más elevada en EPOC (78\%) y cáncer de pulmón (82\%). Asimismo, el $18 \%$ de neumonías y el $25 \%$ de las muertes por otros tipos de cáncer se atribuyen al consumo. 
Tabla 1. Datos demográficos, epidemiológicos, de costos e impositivos incluidos en el modelo (costos expresados en moneda local, 2015).

\begin{tabular}{|c|c|c|c|c|}
\hline Parámetro & Hombres & Mujeres & & Fuente \\
\hline Población $\geq 35$ años (millones) & 5,91 & 6,11 & & (21) \\
\hline \multicolumn{5}{|l|}{$\begin{array}{l}\text { Prevalencia de tabaquismo (porcentaje de la } \\
\text { población) }\end{array}$} \\
\hline $35-49$ años & 30,8 & 25,7 & & $(4)$ \\
\hline 50-64 años & 19,9 & 10,1 & & $(4)$ \\
\hline$\geq 65$ años & 19,9 & 10,1 & & $(4)$ \\
\hline \multicolumn{5}{|l|}{ Tasa de mortalidad cruda (por 10000 habitantes) } \\
\hline Infarto de miocardio & 74,6 & 57,3 & & $(22)$ \\
\hline Otras enfermedades cardiovasculares & 51,8 & 57,2 & & $(22)$ \\
\hline Accidente cerebrovascular & 52,6 & 50,7 & & $(22)$ \\
\hline Neumonía & 220,9 & 199,0 & & $(22)$ \\
\hline Enfermedad Pulmonar Obstructiva Crónica & 33,2 & 25,3 & & $(22)$ \\
\hline Cáncer de pulmón & 13,5 & 10,4 & & $(22)$ \\
\hline Otros tipos de cáncer & 37,2 & 39,0 & & $(22)$ \\
\hline Costos y calidad de vida & $\begin{array}{c}\text { Costo del primer año } \\
\text { o del año del evento } \\
\text { (PEN) }\end{array}$ & $\begin{array}{c}\text { Costo anual de } \\
\text { seguimiento (año dos y } \\
\text { subsiguientes) (PEN) }\end{array}$ & $\begin{array}{l}\text { Ponderación } \\
\text { de cada año de } \\
\text { vida (AVAC) }\end{array}$ & $\begin{array}{l}\text { Fuente } \\
\text { AVAC }\end{array}$ \\
\hline Infarto de miocardio & 9000 & 3959 & 0,8 & $(23)$ \\
\hline Otras enfermedades cardiovasculares & 6252 & 3959 & 0,8 & $(24)$ \\
\hline Accidente cerebrovascular & 17096 & 2715 & $0,641 / 0,74^{*}$ & $(23,25)$ \\
\hline Neumonía & 589 & NR & 0,994 & $(26,27)$ \\
\hline EPOC leve & 493 & NR & 0,935 & $(28)$ \\
\hline EPOC moderado & 1292 & NR & 0,776 & $(28)$ \\
\hline EPOC severo & 14746 & NR & 0,689 & $(28)$ \\
\hline Cáncer de pulmón & 47594 & 61540 & 0,5 & $(29)$ \\
\hline Cáncer de boca & 34268 & 23385 & 0,84 & $(30)$ \\
\hline Cáncer de esófago & 39979 & 27077 & 0,63 & $(31,32)$ \\
\hline Cáncer de estómago & 39027 & 29539 & 0,55 & (33) \\
\hline Cáncer de páncreas & 32364 & 22154 & 0,55 & (34) \\
\hline Cáncer de riñón & 34268 & 24000 & 0,78 & (35) \\
\hline \multicolumn{5}{|l|}{ Datos impositivos } \\
\hline $\begin{array}{l}\text { Ingresos Nacionales por impuestos al } \\
\text { tabaco (millones) }\end{array}$ & 231 & & & $\ddagger$ \\
\hline Producto Interno Bruto (millones) & 576118 & & & $(36)$ \\
\hline Elasticidad de la demanda ${ }^{\dagger}$ & $-0,430$ & & & $(37)$ \\
\hline Gasto total en salud ( $\%$ PIB) & $5,3 \%$ & & & $(36)$ \\
\hline Gasto público en salud (\% del gasto total) & $60,6 \%$ & & & $(36)$ \\
\hline
\end{tabular}

$\mathrm{PEN}=$ soles; $\mathrm{NR}=$ No requerido; $\mathrm{PBI}=$ Producto Interno Bruto

${ }^{*}$ Primer año/subsiguientes.

Tasa de cambio: 1 USD=2,9870 PEN.

† Se asume equivalente al valor promedio reportado. ‡ Estimación de la Comisión Nacional Permanente de Lucha Antitabáquica (COLAT) en base a Superintendencia Nacional de Administración Tributaria (SUNAT).

Anualmente, el tabaquismo es responsable de 95665 eventos. De estos, 4830 corresponden a infartos de miocardio, 2023 a episodios coronarios agudos, 7936 a ACV y 6926 a nuevos diagnósticos de cáncer, siendo la fracción atribuible de $82 \%$ para cáncer de pulmón, de $81 \%$ para cáncer de laringe, de $67 \%$ para cáncer de esófago y de $62 \%$ para cánceres de boca y faringe, teniendo también influencia en el cáncer de estómago, páncreas y riñón (Tabla 2).

En hombres, el $14 \%$ de todas las muertes y el $35 \%$ de los eventos por patologías asociadas se atribuyen al tabaquismo, mientras que en mujeres dicho valor es del 10,4 y $27 \%$ respectivamente. 

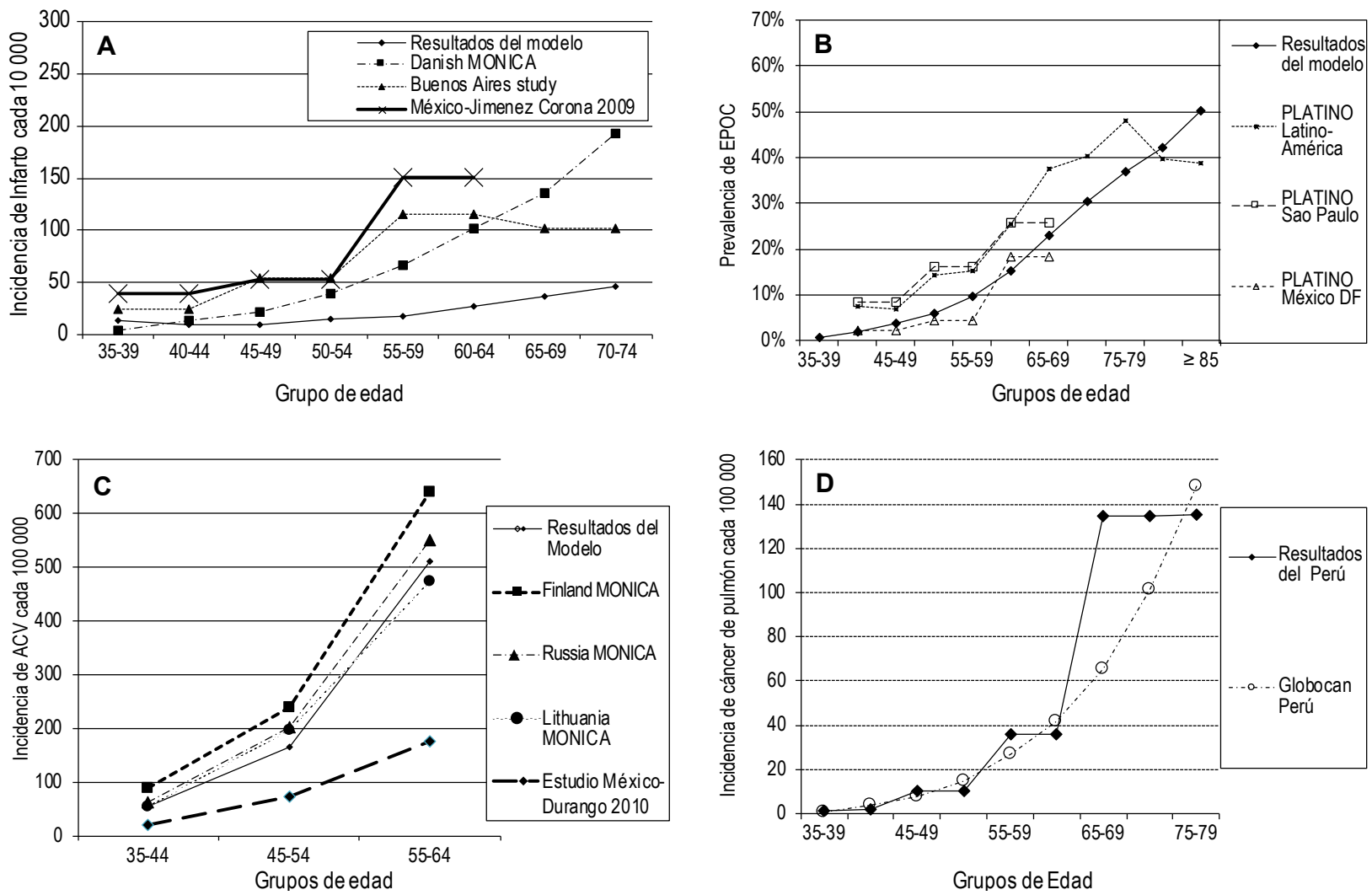

Figura 1. Validación externa del modelo de carga de enfermedad en Perú. A. Incidencia de infarto predicha por el modelo comparado con estudios de incidencia de base poblacional ${ }^{(38-40)}$; B. Prevalencia de EPOC predicha por el modelo comparado con la prevalencia reportada por el estudio Platino ${ }^{(41)}$; C. Incidencia de accidente cerebrovascular (ACV) predicha por el modelo comparado con el WHO MONICA ${ }^{(42)}$; D. Incidencia de cáncer de pulmón predicha por el modelo comparado con las estimaciones de la International Agency for Research on Cancer (IARC) ${ }^{(43)}$.

\section{AÑOS DE VIDA PERDIDOS Y CALIDAD DE VIDA ASOCIADOS AL TABAQUISMO}

La expectativa de vida en hombres fumadores fue 4,7 años menor que los no fumadores, mientras que la reducción en exfumadores fue de 2,1 años. Dicho efecto fue más importante en las mujeres con una expectativa de vida de 5,5 años menor en fumadoras y 2,1 en exfumadoras en relación con las no fumadoras. También en ellas se observó una mayor reducción de la esperanza de vida expresada en AVAC, con una diferencia de 3,5 AVAC entre fumadores y no fumadores en hombres y de 4,1 AVAC en mujeres.

El consumo de tabaco es responsable de 350504 años de vida perdidos (AVP) cada año (232 475 en hombres y 118028 en mujeres), resultante de una combinación de $72 \%$ de AVP por muerte prematura (AVP-mp) y $28,2 \%$ de AVP por vivir en condiciones subóptimas de calidad de vida relacionada a la salud (AVP-cvrs). Si se considerara el tabaquismo pasivo y otras causas (como enfermedad perinatal y accidentes relacionados al tabaquismo) el valor anual ascendería a 396069 AVP.

Los principales responsables de los AVP-mp fueron el cáncer de pulmón $(16,1 \%)$, EPOC $(26,1 \%)$, muerte cardiovascular isquémica $(14,0 \%)$ y accidentes cerebrovasculares $(9,9 \%)$.

\section{COSTOS ASOCIADOS CON EL TABAQUISMO}

El costo directo anual de enfermedades relacionadas con el tabaco ascienden a PEN 6706710 706, de los cuales el 37,8\% (PEN 2535315 893) son atribuibles al tabaquismo (Tabla 2), siendo las enfermedades cardíacas (alta prevalencia) así como los cánceres (principalmente de pulmón) y el EPOC los principales determinantes de su elevado costo.

\section{EFECTO ESPERADO DEL AUMENTO DE PRECIO A TRAVÉS DE IMPUESTOS}

Un aumento de tan solo un $25 \%$ del precio de los cigarrillos podría evitar hasta 6695 muertes en los 
Tabla 2. Muertes, eventos y costos asociados y atribuibles al tabaquismo en Perú, 2015

\begin{tabular}{|c|c|c|c|c|c|c|}
\hline Patología asociada al tabaquismo & $\begin{array}{l}\text { Muertes } \\
\text { totales }\end{array}$ & $\begin{array}{c}\text { Muertes } \\
\text { atribuibles } \\
\text { (\% del total } \\
\text { de patologías } \\
\text { asociadas) }\end{array}$ & $\begin{array}{l}\text { Eventos } \\
\text { totales }\end{array}$ & $\begin{array}{l}\text { Eventos } \\
\text { atribuibles } \\
\text { (\% del total } \\
\text { de eventos } \\
\text { atribuibles) }\end{array}$ & $\begin{array}{c}\text { Costos } \\
\text { totales } \\
\text { (millones de } \\
\text { PEN) }\end{array}$ & $\begin{array}{c}\text { Costos } \\
\text { atribuibles } \\
\text { (millones de } \\
\text { PEN) }\end{array}$ \\
\hline Infarto de miocardio & 6150 & $1214(7,3)$ & 20217 & $4830(23,9)$ & & \\
\hline Síndrome coronario agudo no IAM & 673 & $112(0,7)$ & 9793 & $2023(20,7)$ & 1222 & 313 \\
\hline $\begin{array}{l}\text { Muerte cardiovascular de causa no } \\
\text { isquémica }\end{array}$ & 4956 & $695(4,2)$ & & & & \\
\hline Accidente cerebrovascular & 6792 & $1122(6,7)$ & 47977 & $7936(16,5)$ & 1706 & 305 \\
\hline Cáncer de pulmón & 2791 & $2277(13,6)$ & 2845 & $2322(81,6)$ & 352 & 288 \\
\hline Neumonía & 14934 & $2742(16,4)$ & 128909 & $24442(19,0)$ & 76 & 14 \\
\hline $\begin{array}{l}\text { Enfermedad pulmonar obstructiva } \\
\text { crónica }\end{array}$ & 5074 & $3936(23,5)$ & 69744 & $49508(71,0)$ & 970 & 745 \\
\hline Tipos de cáncer & & & & & 2381 & 578 \\
\hline Cáncer de boca y faringe & 306 & $193(1,2)$ & 767 & $473(61,7)$ & & \\
\hline Cáncer de esófago & 404 & $274(1,6)$ & 439 & $296(67,4)$ & & \\
\hline Cáncer de estómago & 5535 & $1161(6,9)$ & 8038 & $1667(20,7)$ & & \\
\hline Cáncer de páncreas & 1255 & $299(1,8)$ & 1323 & $317(24,0)$ & & \\
\hline Cáncer de riñón & 570 & $167(1,0)$ & 1172 & $339(28,9)$ & & \\
\hline Cáncer de laringe & 214 & $172(1,0)$ & 469 & $378(80,6)$ & & \\
\hline Leucemia & 827 & $136(0,8)$ & 893 & $149(16,7)$ & & \\
\hline Cáncer de vejiga & 357 & $148(0,9)$ & 1425 & $584(41,0)$ & & \\
\hline Cáncer de cuello de útero & 1539 & $149(0,9)$ & 4055 & $401(9,9)$ & & \\
\hline Tabaquismo pasivo y otras causas & 1923 & $1923(11,5)$ & & & & \\
\hline $\begin{array}{l}\text { Total por patologías asociadas al } \\
\text { tabaquismo }\end{array}$ & 54301 & 16719 & 298066 & 95665 & 6706 & 2535 \\
\hline
\end{tabular}

$\mathrm{PEN}=$ soles

Tasa de cambio: 1 USD=2,9870 PEN

próximos diez años y reportar importantes beneficios económicos (Tabla 3). Ante un escenario de incremento en $50 \%$ del precio de cigarrillos por carga impositiva se podrían evitar 13391 muertes, 320584 AVP y 23549 eventos. Asimismo, se tendría un beneficio económico de más de PEN 3,1 mil millones en diez años. Estos resultados llegan, en algunos casos, a duplicarse ante un incremento del $100 \%$.

Tabla 3. Impacto en la salud, costos sanitarios y recaudación impositiva a diez años. Tres escenarios de aumento de precio de cigarrillos a través de impuestos en Perú, 2015.

\begin{tabular}{lccc}
\hline \multirow{2}{*}{ Parámetro } & \multicolumn{3}{c}{ Aumento de precio } \\
\cline { 2 - 4 } & $\mathbf{2 5 \%}$ & $\mathbf{5 0 \%}$ & $\mathbf{1 0 0 \%}$ \\
\hline Muertes evitadas & 6695 & 13391 & 26782 \\
\hline Enfermedades cardíacas evitadas & 3105 & 6210 & 12420 \\
\hline Accidentes cerebrovasculares evitados & 5989 & 11978 & 23957 \\
Nuevos casos de cáncer evitados & 2680 & 5361 & 10721 \\
Años de vida debidos a muerte prematura y discapacidad evitados & 160292 & 320584 & 641168 \\
\hline Costos sanitarios evitados (millones PEN) & 1027 & 2054 & 4108 \\
Aumento en la recaudación impositiva (millones PEN) & 641 & 1090 & 1419 \\
\hline Beneficio económico total (millones PEN) & 1668 & 3145 & 5527 \\
\hline
\end{tabular}

$\mathrm{PEN}=$ soles

Tasa de cambio: 1 USD=2,9870 PEN 
La Figura 2 muestra el escenario de aumento de precio de $50 \%$, y como los beneficios en mortalidad y en costos se van incrementando a lo largo de los años, evidenciándose una importante reducción acumulada de las muertes y costos sanitarios.
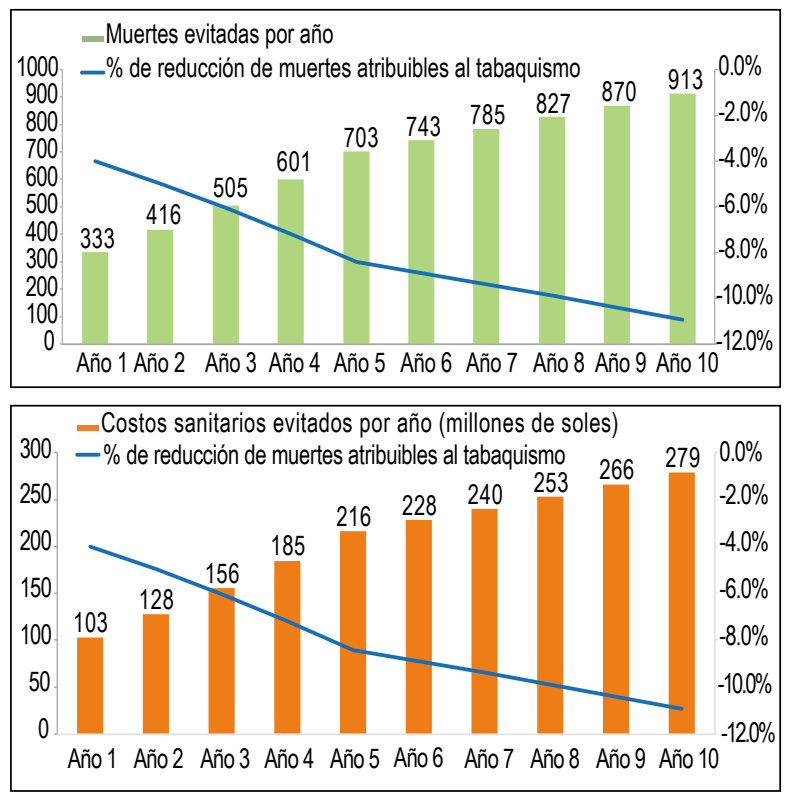

Figura 2. Escenario de aumento de precio de cigarrillos de un 50\%. Beneficios esperados a diez años en Perú, 2015.

\section{DISCUSIÓN}

Nuestro estudio estima que en Perú, el tabaquismo genera, por un lado, una carga sanitaria importante, expresada en muertes, enfermedades, disminución de calidad de vida y, por otro, un gran costo para el sistema de salud, con una mayor carga proveniente de las enfermedades cardiovasculares, EPOC y el cáncer de pulmón. Es responsable del $12,5 \%$ de todas las muertes evitables que se producen en el país en mayores de 35 años (16 719 muertes por año) y genera una pérdida directa de más de 110 mil años de vida saludable cada año. Asimismo, agrega un costo directo por más de PEN 2535 millones/ año, cifra que representa el $0,4 \%$ del producto bruto interno (PBI) y el 7,8\% del gasto total en salud.

La recaudación tributaria en el 2015 por comercio de cigarrillos en el Perú, representa un aproximado de PEN 231 millones, sin embargo, esta cifra, se encuentra muy por debajo del gasto directo ocasionado por el tabaco. Una estrategia razonable y utilizada en otros países, seria un incremento en $50 \%$ del precio de cigarrillos a través de los impuestos, esto podría no solamente prevenir las muertes y eventos, sino, además, lograr un beneficio económico de más de PEN 3,1 mil millones en diez años. Dicho incremento sería consistente con numerosos estudios que reportan resultados similares en términos de carga global y en la reducción potencial del consumo a través de los impuestos ${ }^{(3,7,44)}$, así como también, estaría aún por debajo del $65 \%$ del precio de venta recomendado por el Banco Mundial; de los dos tercios o cuatro quintos que se impone en países de altos ingresos y con precios por cajetilla equiparados con países de la región ${ }^{(11,45)}$.

Es importante notar que esta estimación puede considerarse conservadora, hemos utilizado un valor medio para la elasticidad precio de la demanda de $-0,43$ proveniente de una revisión para Latinoamérica. No obstante, es altamente probable que este valor sea mayor en ciertos subgrupos (como podrían ser mujeres o jóvenes) lo cual aumentaría el impacto beneficioso que podría lograrse mediante un aumento en el precio.

En el Perú, el tabaquismo es la segunda droga lícita más consumida, y uno de los principales factores de riesgo para enfermedades crónicas; convirtiéndose así en un problema de salud pública ${ }^{(46)}$. Una encuesta mostró un mayor consumo en participantes con nivel de educación superior $(65,0 \%)$, seguido por aquellos con nivel secundario $(52,5 \%)$ y primario $(43,3 \%)$, con una edad promedio de iniciación de 17,5 años y una mayor prevalencia (64,3\%) en el grupo de 19 a 25 años ${ }^{(47)}$, es decir, el tabaquismo predomina en población joven. En ese sentido, un estudio de varios países, incluido Perú, evaluó el impacto de los precios en la iniciación y cesación del consumo en jóvenes, encontrando que precios más altos retrasan únicamente la iniciación ${ }^{(48)}$. Por su parte, el Banco Mundial considera que el incremento de impuestos decrece el consumo especialmente de jóvenes en $7 \%$ con incremento del $10 \%$ del precio de cigarrillos ${ }^{(45)}$. Igualmente, se estima que en los países de más altos ingresos en América Latina un aumento del $10 \%$ en los precios reduciría la demanda de cigarrillos en al menos $5 \%{ }^{(37)}$. Los efectos negativos que trae consigo el consumo temprano de tabaco, convierte al tabaquismo en un grave problema para la salud pública, que debería ser abordado rápida y eficazmente para reducir su impacto en lo personal, económico y social.

Según un estudio del Ministerio de Salud del Perú, en el 2012 se perdieron 5800949 años saludables (AVISA) por enfermedades y lesiones ${ }^{(49)}$. La mayor carga de enfermedad se atribuyó a las enfermedades no transmisibles $(60,5 \%)$. Nuestro estudio encontró que el aumento del impuesto al tabaco disminuye la cantidad de muertes y eventos relacionados al tabaquismo. Resultados similares fueron encontrados en un estudio en Argentina basado en elasticidad precio-demanda, el cual sugiere que un incremento del $100 \%$ en el precio final de cigarrillo podría disminuir el consumo en un $31 \%$ y evitar más de 15000 muertes por eventos coronarios en un periodo de diez años ${ }^{(50)}$. 
Ante este escenario, el país debe disponer de recursos que le permitan proveer los servicios de salud que necesita su población para garantizar un adecuado nivel de salud. Es así que las evaluaciones basadas en modelos económicos de salud son ampliamente aceptadas como herramientas para la toma de decisiones y pueden proporcionar información valiosa en la búsqueda de optimizar la asignación de recursos sanitarios. Para tal efecto, nuestros resultados muestran que un incremento en el precio de los cigarrillos contribuiría en el largo plazo a desincentivar su consumo, generando, por un lado, una disminución de la carga de enfermedad y por otro, la recaudación de mayores recursos que podrían ser canalizados hacia la implementación de programas o políticas de lucha contra el tabaco en el Perú.

Este es uno de los primeros estudios en Latinoamérica en usar un modelo de decisión con sólida evidencia de la literatura y datos validados por expertos de diferentes países (12); no obstante, presenta algunas limitaciones. Por un lado, se trata de una estimación conservadora, debido a la falta de inclusión de costos por ausentismo laboral y productividad perdida, gastos de bolsillo y otras consecuencias sobre la economía del hogar que podría, incluso, triplicar la carga económica ${ }^{(3,51)}$. Por otro lado, aunque impactan relativamente poco en las estimaciones totales, podría mejorarse la metodología estimando la carga de enfermedad por tabaquismo pasivo y efectos perinatales. Asimismo, sería de interés explorar la carga de enfermedad en diversas subpoblaciones, por ejemplo, debido a las diferentes elasticidades precio de la demanda entre hombres y mujeres, jóvenes/ adolescentes y adultos, entre otros. Adicionalmente, existió escasa información sobre incidencias; sin embargo, al tratarse de datos importantes para nuestro modelo, fueron estimadas a partir de registros y/o estadísticas nacionales.

A pesar de que la evidencia es clara respecto a los beneficios del aumento de impuestos al tabaco, muchos países no han logrado aún implementar o profundizar suficientemente esta medida, principalmente por la falta de evidencia local que podría generar incertidumbre en los decisores sobre el potencial impacto de esta medida. Asimismo, los resultados están alineados a otros estudios que han demostrado escenarios económicos, de salud y laborales favorables ante este tipo de medidas ${ }^{(44,52)}$. Por lo tanto, este estudio se convierte en un medio de soporte para futuras acciones por parte de los decisores en la implementación de medidas orientadas al control del tabaquismo y, a la vez, provee elementos locales válidos para el desarrollo de futuras investigaciones.

En conclusión, el tabaquismo es responsable de una alta carga de enfermedad en Perú y consume una importante proporción de los recursos sanitarios disponibles en el país. Un aumento de los impuestos al cigarrillo -que actualmente tiene un precio bajo en relación a muchos vecinos de la región- podría tener importantes beneficios económicos y de salud. En tal sentido, es esperable que los resultados de este estudio contribuyan a tomar conciencia sobre los efectos del tabaco en Perú y sean un soporte para que las autoridades gubernamentales y otros actores de la sociedad puedan lograr implementar esta política tan costo-efectiva.

Agradecimientos: a las Dras. Brunilda Casetta y Ruth Ruano Gándara, de Argentina, por su ayuda en la evaluación del contenido y al Lic. Daniel Comandé, bibliotecario del Instituto de Efectividad Clínica y Sanitaria por su ayuda con las búsquedas bibliográficas.

Contribuciones de autoría: $A P R, F A, A B, A A, J C$ fueron responsables de la idea general y de la conducción del proyecto en varios países. JC, $A A, A B$ recopilaron los datos epidemiológicos y de costos. LHF, CLM aportaron datos primarios, ayudaron en la redacción de diferentes secciones, AB, JC, AA, FA, LHF, CLM, AHV y APR analizaron e interpretaron los datos, redactaron el manuscrito y aprobaron la versión final.

Fuentes de financiamiento: el proyecto fue financiado mediante un subsidio de investigación otorgado por el Centro Internacional de Investigación para el Desarrollo de Canadá (IDRC).

Declaración de conflictos de interés: los autores expresan no poseer conflictos de interés.

\section{REFERENCIAS BIBLIOGRÁFICAS}

1. Lim SS, Vos T, Flaxman AD, Danaei G, Shibuya K, Adair-Rohani H, et al. A comparative risk assessment of burden of disease and injury attributable to 67 risk factors and risk factor clusters in 21 regions, 1990-2010: a systematic analysis for the Global Burden of Disease Study 2010. Lancet. 2012;380(9859):2224-60.

2. Ciapponi A, Bardach A, Casetta B, Aruj P, Linetzky B, Glujovsky D, et al. Tobacco
Free Initiative. Systematic review of thelink between tobacco and poverty. Research commissioned for WHO by the Institute for Clinical Effectiveness and Health Policy (Instituto de Efectividad Clínica y Sanitaria -IECS), Argentine Cochrane Centre IECS, Iberoamerican Cochrane Network. WHO Library Cataloguingin-Publication Data; 2011 [citado el 2 de febrero de 2015]. Disponible en: http:// www.who.int/tobacco/publications/syst_ rev_tobacco_poverty/en/index.html. 2010.

3. Lightwood J, Lapsley H, Novotny T. Estimating the costs of tobacco use. In: Jha P, Chaloupka F, editors. Tobacco control in developing countries Oxford: Oxford University Press; 2000. p. 63-99.

4. Chávez Rodas V, Tapia Cabanillas L, Rojas Valero M, Zavaleta Martinez- 
Vargas A. Epidemiología de drogas en la población urbana peruana 2010. Encuesta de Hogares. Lima: CEDRO; 2011.

5. Weygandt PL, Vidal-Cardenas E, Gilman RH, Avila-Tang E, Cabrera L, Checkley W. Epidemiology of tobacco use and dependence in adults in a poor peri-urban community in Lima, Peru. BMC Pulm Med. 2012;12:9.

6. Organización Mundial de la Salud (OMS). Partes en el Convenio Marco de la OMS para el Control del Tabaco [Internet]. 2015 [citado el 11 de noviembre de 2015]. Disponible en: http://www.who.int/fctc/signatories_ parties/es

7. Hoffman SJ, Tan C. Overview of systematic reviews on the healthrelated effects of government tobacco control policies. BMC public health. 2015;15:744

8. Tauras JA, Chaloupka FJ. The demand for nicotine replacement therapies [Internet]. United States. National Bureau of Economic Research; 2001 [citado el 15 de febrero de 2016]. Disponible en: http:// www.nber.org/papers/w8332.

9. Guindon GE, Tobin S, Yach D. Trends and affordability of cigarette prices: ample room for tax increases and related health gains. Tobacco control. 2002;11(1):35-43.

10. Chaloupka F, Hu T-W, Warner K, Jacobs R, Yurekli A. The taxation of tobacco products. In: Tobacco control in developing countries [Internet]. Oxford: Oxford University Press; 2000. Disponible en: http:// catalogue.polytechnique.fr/site. php id $=298 \&$ fileid $=4705$.

11. World Health Organization. Report on the global tobacco epidemic 2015. Appendix IX: Tax and price data [Internet]. [citado el 22 de enero de 2016]. Disponible en: http:// www.who.int/tobacco/global_ report/2015/appendix_ix/en

12. Pichon-Riviere A, Augustovski F, Bardach A, Colantonio L. Development and validation of a microsimulation economic model to evaluate the disease burden associated with smoking and the cost-effectiveness of tobacco control interventions in Latin America. Value Health 2011;14(5 Suppl 1):S51-9.

13. McLean S, Barbour V, Wild S, Simpson C, Sheikh A. Models for estimating projections for disease prevalence and burden: a systematic review focusing on chronic obstructive pulmonary disease. Journal of health services research \& policy. 2015;20(4):246-53.

14. Pinto MT, Pichon-Riviere A, Bardach A. The burden of smoking-related diseases in Brazil: mortality, morbidity and costs. Cad Saude Publica. 2015;31(6):1283-97.

15. Pichon-Riviere A, Alcaraz A, Bardach A, Augustovski F, Caporale J, Caccavo Francisco. Carga de Enfermedad atribuible al Tabaquismo en Argentina. Documento Técnico IECS $\mathrm{N}^{\circ} 7$ [Internet]. Buenos Aires, Argentina: Instituto de Efectividad Clínica y Sanitaria; 2013 [citado el 25 de enero de 2016]. Disponible en: http://www. iecs.org.ar/publicacion $/$ ?id= 5471

16. Pichón Riviere A, Bardach A, Caporale J, Alcaraz A, Augustovski F, Caccavo F, et al. Carga de Enfermedad atribuible al Tabaquismo en Chile. Documento Técnico IECS $\mathrm{N}^{\circ} 8$ [Internet]. Buenos Aires, Argentina: Instituto de Efectividad Clínica y Sanitaria; 2014 [citado el 25 de enero de 2016]. Disponible en: http://www.iecs.org. $\mathrm{ar} /$ publicacion $/ \mathrm{id}=5472$

17. Pichon-Riviere A, ReynalesShigematsu LM, Bardach A, Caporale J, Augustovski F, Alcaraz A, et al. Carga de Enfermedad Atribuible al Tabaquismo en México. Documento Técnico IECS $\mathrm{N}^{\circ} 10$ [Internet]. Buenos Aires, Argentina: Instituto de Efectividad Clínica y Sanitaria; 2013 [citado el 25 de enero de 2016]. Disponible en: http://www.iecs.org. ar/publicacion $/$ id $=5473$

18. Centers for Disease Control and Prevention (CDC). Smokingattributable mortality, years of potential life lost, and productivity losses. United States, 2000-2004. MMWR Morb Mortal Wkly Rep. 2008;57(45):1226-8.

19. Doll R, Peto R, Boreham J, Sutherland I. Mortality in relation to smoking: 50 years' observations on male British doctors. BMJ (Clinical research ed). 2004;328(7455):1519.
20. World Health Organization. International Agency for Research on Cancer. Tobacco Smoke and Involuntary Smoking [Internet]. Lyon: France; 2005 [citado el 11 de febrero de 2015]. Disponible en: http://monographs.iarc.fr/ENG/ Monographs/vol83/mono83-1.pdf.

21. Estimaciones y proyecciones de población, 1950-2050 [Internet]. Lima: Instituto Nacional de Estadística e Informática; 2001 [citado el 15 de febrero de 2015]. Disponible en http://www.inei.gob.pe/media/ MenuRecursivo/publicaciones digitales/Est/Lib0466/Libro.pdf

22. Oficina General de Estadística e Informática [Internet]. Lima: Ministerio de Salud; 2010 [citado el 20 de febrero 2015]. http://www. minsa.gob.pe/portada/estadistica/ publicaciones.htm

23. Main C, Palmer S, Griffin S, Jones L, Orton V, Sculpher M, et al. Clopidogrel used in combination with aspirin compared with aspirin alone in the treatment of non-ST-segmentelevation acute coronary syndromes: a systematic review and economic evaluation. Health technology assessment (Winchester, England). 2004;8(40):iii-iv, xv-xvi, 1-141.

24. Nease RF Jr, Kneeland T, O'Connor GT, Sumner W, Lumpkins C, Shaw $\mathrm{L}$, et al. Variation in patient utilities for outcomes of the management of chronic stable angina. Implications for clinical practice guidelines. Ischemic Heart Disease Patient Outcomes Research Team. Jama. 1995;273(15):1185-90.

25. Tengs TO, Yu M, Luistro E. Healthrelated quality of life after stroke a comprehensive review. Stroke; a journal of cerebral circulation. 2001;32(4):964-72.

26. Pepper PV, Owens DK. Costeffectiveness of the pneumococcal vaccine in healthy younger adults. Med Decis Making. 2002;22(5 Suppl):S45-57.

27. Hamel MB, Phillips RS, Davis RB, Teno J, Connors AF, Desbiens N, et al. Outcomes and cost-effectiveness of ventilator support and aggressive care for patients with acute respiratory 
failure due to pneumonia or acute respiratory distress syndrome. Am J Med. 2000;109(8):614-20.

28. Rutten-van Molken M, Lee TA. Economic modeling in chronic obstructive pulmonary disease. Proc Am Thorac Soc. 2006;3(7):630-4.

29. Montazeri A, Gillis CR, McEwen J. Quality of life in patients with lung cancer: a review of literature from 1970 to 1995 . Chest. $1998 ; 113(2): 467-81$.

30. Downer MC, Jullien JA, Speight PM. An interim determination of health gain from oral cancer and precancer screening: 1. Obtaining health state utilities. Community dental health. 1997;14(3):139-42.

31. Graham AJ, Shrive FM, Ghali WA, Manns BJ, Grondin SC, Finley RJ, et al. Defining the optimal treatment of locally advanced esophageal cancer: a systematic review and decision analysis. Ann Thorac Surg. 2007;83(4):1257-64.

32. Shenfine J, McNamee P, Steen N, Bond J, Griffin SM. A pragmatic randomised controlled trial of the cost-effectiveness of palliative therapies for patients with inoperable oesophageal cancer. Health technology assessment (Winchester, England). 2005;9(5):iii, 1-121.

33. Dan YY, So JB, Yeoh KG. Endoscopic screening for gastric cancer. Clin Gastroenterol Hepatol. 2006;4(6):709-16.

34. Gordois A, Scuffham P, Warren E, Ward S. Cost-utility analysis of imatinib mesilate for the treatment of advanced stage chronic myeloid leukaemia. $\mathrm{Br} \mathrm{J}$ Cancer. 2003;89(4):634-40.

35. Heinzer H, Mir TS, Huland E, Huland H. Subjective and objective prospective, long-term analysis of quality of life during inhaled interleukin-2 immunotherapy. J Clin Oncol. 1999;17(11):3612-20.

36. World Development Indicators, Data [Internet]. The World Bank; 2015. [citado el 13 de noviembre de 2015]. Disponible en: http://data. worldbank.org/data-catalog/worlddevelopment-indicators.

37. Guindon GE, Paraje GR, Chaloupka FJ. The impact of prices and taxes on the use of tobacco products in Latin America and the Caribbean. Am J Public Health 2015;105(3):e9-19.

38. Kirchhoff M, Davidsen M, BronnumHansen H, Hansen B, Schnack $\mathrm{H}$, Eriksen LS, et al. Incidence of myocardial infarction in the Danish MONICA population 1982-1991. Int J Epidemiol. 1999;28(2):211-8.

39. Caccavo A, Alvarez A, Bello F, et al. Incidencia poblacional del infarto con elevación del segmento ST o bloqueo de rama izquierda a lo largo de 11 años en una comunidad de la provincia de Buenos Aires. Rev Argent Cardiol. 2007;75:185-8.

40. Jiménez-Corona A, López-Ridaura R, González-Villalpando C. Incidence of myocardial infarction in low-income urban residents of Mexico City. Salud pública Méx. 2009;51:458-64.

41. Menezes AM, Perez-Padilla R, Jardim JR, Muino A, Lopez MV, Valdivia G, et al. Chronic obstructive pulmonary disease in five Latin American cities (the PLATINO study): a prevalence study. Lancet. 2005;366(9500):1875-81.

42. Thorvaldsen P, Asplund K, Kuulasmaa K, Rajakangas AM, Schroll M. Stroke incidence, case fatality, and mortality in the WHO MONICA project. World Health Organization Monitoring Trends and Determinants in Cardiovascular Disease. Stroke. 1995;26(3):361-7.

43. Ferlay J, Soerjomataram I, Ervik M, Dikshit R, Eser S, Mathers C, et al. GLOBOCAN 2012 v1.0, Cancer Incidence and Mortality Worldwide: IARC CancerBase No. 11 [Internet]. Lyon, France: International Agency for Research on Cancer; 2013 [citado el 15 de enero del 2016. Disponible en: http://globocan.iarc.fr.

44. Chaloupka FJ, Yurekli A, Fong GT. Tobacco taxes as a tobacco control strategy. Tob control. 2012;21(2):172-80.

45. Ekpu VU, Brown AK. The Economic Impact of Smoking and of Reducing Smoking Prevalence: Review of Evidence. Tobacco use insights. 2015;8:1-35.
46. Dirección General de Epidemiología. Epidemiología del consumo de tabaco en el Perú. Boletin Epidemiológico (Lima) 23(2) [Internet]. Lima: Ministerio de Salud; 2014 [citado el 25 de enero del 2016]. Disponible en: http://www.dge.gob.pe/portal/docs/ vigilancia/boletines/2014/02.pdf.

47. Impacto social y económico del consumo de drogas en el Perú. Lima; DEVIDA; 2010.

48. Kostova D. A (nearly) global look at the dynamics of youth smoking initiation and cessation: the role of cigarette prices. Applied Economics. 2013;45(28):3943-51.

49. Dirección General de Epidemiología. Carga de enfermedad en el Perú. Estimación de los años de vida saludables perdidos 2012 [Internet]. Lima: Ministerio de Salud; 2014 [citado el 15 de enero de 2016]. Disponible en: http://www. dge.gob.pe/portal/docs/tools/ Cargaenfermedad2012.pdf.

50. Martinez E, Mejia R, Perez-Stable EJ. An empirical analysis of cigarette demand in Argentina. Tob control. 2015;24(1):89-93.

51. Bundhamcharoen K, Aungkulanon S, Makka N, Shibuya K. Economic burden from smoking-related diseases in Thailand. Tob control. 2015.

52. Ho V, Ross JS, Steiner CA, Mandawat A, Short $\mathrm{M}, \mathrm{Ku}$-Goto $\mathrm{MH}$, et al. A Nationwide Assessment of the Association of Smoking Bans and Cigarette Taxes With Hospitalizations for Acute Myocardial Infarction, Heart Failure, and Pneumonia. Med Care Res Rev. 2016. pii: 1077558716668646. [Epub ahead of print]

Correspondencia: Ariel Bardach Dirección: Instituto de Efectividad Clínica y Sanitaria. Dr. Emilio Ravignani 2024 (C1414CPV), Ciudad Autónoma de Buenos Aires. Buenos Aires, Argentina.

Teléfono: (005411) 47778767

Correo electrónico:abardach@iecs.org.ar 